\title{
Bibliografía mexicana de arte
}

\section{7}

\author{
por \\ XAVIER MOYSSÉN
}

No obstante la crisis económica por la que atraviesa el país, la producción de libros de arte durante 1997 fue de cierta consideración. Se publicaron obras de atractiva calidad en sus impresiones pero, sobre todo, de una relevante importancia por los estudios que contienen; son interesantes aportaciones a la historia del arte. Sobresalen como editores la U niversidad $\mathrm{N}$ acional Autónoma de M éxico, el Consejo $\mathrm{N}$ acional para la $\mathrm{Cul-}$ tura y las Artes, institución que ha lanzado una colección de libros dedicados a distintos aspectos de la plástica, titulada "Círculo de Arte"; la colección cuenta ya con veinte títulos publicados. Por su labor como editoras sobresalen algunas instituciones bancarias,

\section{Artes plásticas y arquitectura. Época prehispánica}

C abrero G., M aría T eresa, C atálogo de piezas de las tumbas de tiro del cañón de Bolaños. M éxico, Universidad $\mathrm{N}$ acional Autónoma de M éxico (Instituto de Investigaciones Antropológicas), 1997.

Coe, M ichael D., The Art of the M aya Scribe. Londres, Thames and H udson, 1997.

$M$ aclaury, Robert E., Color and Cognition in M esoamerica: Constructing Categories as Vantages. Austin, University of T exas, 1997.

M edina, M iguel Ángel, Arte y estética de El las cuales cuentan en su haber con un número crecido de títulos publicados en el transcurso de varios años.

En la bibliografía anual dedicada al arte, indudable importancia es la que tienen los catálogos de las exposiciones de artes plásticas presentadas tanto en museos como en galerías; los catálogos son imprescindibles como documentos por los estudios que contienen, debidos en la mayoría de los casos a autores que son expertos en los temas de que se ocupan. Las reproducciones de las obras presentadas en las exposiciones adquieren el valor documental que en el futuro los investigadores encontrarán en ellas.

T etzcocingo. La arquitectura de paisaje en la época de $\mathrm{N}$ etzahualcóyotl. M éxico, Universidad $\mathrm{N}$ acional Autónoma de $M$ éxico (Coordinación de $\mathrm{H}$ umanidades), 1997 (Colección de Arte, 50).

Oliver Vega, Beatriz, Papel ceremonial entre los otomíes. Presentación de M ari Carmen Serra Puche, M éxico, Instituto $\mathrm{N}$ acional de Antropología e H istoria, 1997.

Ortega, Ana, Pintura mural mesoamericana. $\mathrm{M}$ éxico, Consejo $\mathrm{N}$ acional para la $\mathrm{Cul-}$ tura y las Artes, 1997 (C írculo de Arte).

Pasztory, Esther, Teotihuacan: An Experiment in Living. Norman, U niversity of O klahoma, I997. 
DOI: http://dx.doi.org/10.22201/iie.18703062e.1998.72.1801

Ramírez H errera, Rogelio, H umano-felino. Ensayos de arqueología veracruzana. Presentación de Sara Ladrón de Guevara, dibujos de Adrián G arcía García, Vera cruz, Instituto Veracruzano de Cultura, I997.

Refless de la vie: Art de l'O ccident du M exique. Catálogo de la exposición presentada en el $\mathrm{H}$ all Charles V, Carrousel du Louvre, texto de 0 tto Schöndube, París, [editor], 1997.

Schele, Linda, Rostros ocultos de los mayas. Introducción de Román Piña Chan, fotografías de J orge Pérez de Lara, M éxico, Ímpetus Comunicación, 1997.

Stierlin, H enri, The M aya: Palaces and Pyramids of the Rainforest. Koln, Taschen, 1997.

\section{Artes plásticas y arquitectura. Época colonial}

Alejandro de $\mathrm{H}$ umboldt en M éxico. M éxico, Instituto $\mathrm{N}$ acional de Antropología e $\mathrm{H}$ istoria, 1997.

Amores prohibidos, La palabra condenada en el M éxico de los virreyes. Antología de coplas y versos censurados en la Inquisición de M éxico. Compilación de Georges Baudot, M éxico, Siglo Veintiuno, 1997.

Ángeles, Pedro, Clara Bargellini, Juana Gutiérrez $\mathrm{H}$ aces y Rogelio Ruiz $\mathrm{Gomar}$, Cristóbal de Villalpando ca. I649-I7I4. Catálogo razonado. M éxico, Fundación Cultural Banamex-U niversidad $\mathrm{N}$ acional Autónoma de M éxico (Instituto de Investigaciones Estéticas), 1997.

Bargellini, Clara, M isiones y presidios de Chihuahua. M éxico, Gobierno del Estado de Chihuahua, 1997.
Cortés Castellanos, Justino, El catecismo en pictogramas de fray Pedro de $\mathrm{G}$ ante. $\mathrm{M} \mathrm{a}$ drid, Fundación U niversitaria Española, 1997.

Dávalos, M arcela, Basura e ilustración. La imprenta de la ciudad de $\mathrm{M}$ éxico a fines de siglo xviII. M éxico, Instituto $\mathrm{N}$ acional de Antropología e H istoria, 1997.

Gómez M artínez, Javier, Fortalezas mendicantes Claves y procesos en los conventos novohispanos del siglo xVI. M éxico, Universidad I beroamericana (D epartamento de Arte), 1997.

- - , H istoricismos de la arquitectura barroca novohispana. M éxico, U niversidad Iberoamericana (D epartamento de Arte), 1997.

González $\mathrm{G}$ alván, M anuel, Voces del barroco en Santa Prisca de Taxco. M éxico, Jaime Salcido y Romo Editor, 1997.

H ernández Franyuti, Regina, I gnacio Caste ra: arquitecto y urbanista de la ciudad de M éxico, I777-I8II. M éxico, Instituto de Investigaciones D r. José M aría Luis M ora, 1997 .

$\mathrm{H}$ istoria de la arquitectura y el urbanismo mexicanos, vol. II, El periodo virreinal, tomo I, El encuentro de dos universos culturales. Compilación y textos de Carlos Chanfón O Imos, M éxico, U niversidad $\mathrm{N}$ acional Autónoma de M éxico-Fondo de Cultura Económica, 1997.

Jiménez Codinach, Guadalupe, M éxico su tiempo de nacer, I750-I82I. Catálogo de exposición, M éxico, Fomento Cultural Banamex, 1997.

J udas, exequias, arcángeles y comedias. Puebla en el siglo XVIII, rescate documental. Compilación de M argarita Peña M uñoz, M é xico, U niversidad Autónoma de Puebla, 1997.

Kuwayama, George, Chinese Cramics in Colo- 
nial M exico. Los Angeles-H onolulu, LoS Angeles County M useum of Art, 1997.

M orales Bocardo, R afael, El convento de San Francisco de San Luis Potosí. Casa capitular de la provincia de Zacatecas. San Luis Potosí, Archivo H istórico del Estado de San Luis Potosí, 1997.

M oreno, Salvador, Ángeles músicos. H ome naje a sor Juana I nés de la Cruz. M éxico, M azapanes T oledo, 1997.

M ullen, Robert J., Architecture and its Sculpture in Viceregal M exico. Austin, U niversity of T exas, 1997.

Perry, Richard D., Blue Lakes and Silver Cities: The Colonial Arts and Architecture of W est M exico. Santa Barbara (California), Espadaña, 1997.

Ramos M edina, M anuel, M ísticas y descalzas. Fundaciones femeninas carmelitas en la N ueva España. M éxico, Centro de Estudios de $\mathrm{H}$ istoria de $\mathrm{M}$ éxico $\mathrm{Condu}$ mex, 1997.

Razo Zaragoza, José Luis, N ombres de ciudades, villas y pueblos del estado de Jalisco en la época virreinal. M éxico, Instituto Jalisciense de Cultura, 1997.

Soberón, Arturo, Cristóbal de Villalpando, el pincel bien temperado. M éxico, Consejo N acional para la Cultura y las Artes, 1997 (C írculo de Arte).

Stella M arco, M argarita, M arfiles de las provincias ultramarinas orientales de España y Portugal. M onterrey, M useo M arco, 1997.

Terán Elizondo, M aría I sabel, Los recursos de la persuasión. La portentosa vida de la muerte de fray J oaquín Bolaños. Zamora, El Colegio de M ichoacán, I997.
Artes plásticas y arquitectura. Época moderna

Carrillo, Charles M., H ispanic N ew M exican Pottery: Evidence of Craft Specialization, I790-I890. Albuquerque, LPD, 1997.

La Casa Chata de Tlalpan. Semblanza de una vieja casa de campo. M éxico, Centro de Investigaciones y Estudios Superiores en Antropología Social, 1997.

Crónica de M éxico. Estampas mexicanas del siglo XIX. M éxico, M useo Amparo, 1997.

El escenario urbano de Pedro G ualdi, I8081857. Catálogo de exposición, M éxico, Instituto $\mathrm{N}$ acional de Bellas Artes (M useo $\mathrm{N}$ acional de Arte)-Consejo $\mathrm{N}$ acional para la Cultura y las Artes, 1997.

Las glorias del Teatro Colón. M éxico, Club de Banqueros de M éxico, 1997.

M oyssén, Xavier, José M aría V elasco. EI paisajista. M éxico, Consejo $\mathrm{N}$ acional para la Cultura y las Artes, 1997 (Círculo de Arte).

Rodríguez, M aricela, Julio Ruelas, una obra en el límite del hastío. M éxico, Consejo $\mathrm{N}$ acional para la Cultura y las Artes, 1997 (C írculo de Arte).

Rodríguez Prampolini, Ida, La crítica de arte en M éxico en el siglo XIX. Estudios y documentos. M éxico, U niversidad $\mathrm{N}$ acional Autónoma de M éxico (Instituto de Investigaciones Estéticas), I997, 3 vols.

\section{Artes plásticas y arquitectura. É poca contemporánea}

Andrade, Lourdes, Arquitectura vegetal. M é xico, Artes de M éxico, 1997 (Libros de la Espiral).

Antonio Seguí. H ombre de ciudades Catálo- 
go de la exposición del Museo Rufino Tamayo, M éxico, Embajada de la República Argentina-Consejo $\mathrm{N}$ acional para la Cultura y las Artes-Instituto $\mathrm{N}$ acional de Bellas Artes, 1997.

Arquitectos mexicanos: entre la tradición y la modernidad. Edición de Fernando de $\mathrm{H}$ aro Lebrija y $\mathrm{O}$ mar Fuentes Elizondo, M éxico, Attame Ediciones, 1997.

Art déco. Un país nacionalista, un M éxico cosmopolita. Catálogo de la exposición del M useo $\mathrm{N}$ acional de Arte, texto de Enrique X. de Anda Alanís, M éxico, Instituto $\mathrm{N}$ acional de Bellas Artes (M useo $N$ acional de Arte), 1997.

Aś etá la cosa. Instalación y arte objeto en América Latina. M éxico, Centro Cultural de Arte Contemporáneo, 1997.

Aver, G erhard, M athias Goeritz. El Eco. Braunschweig (Alemania), 1997.

¡Cámara! Ciudad de M éxico. M onumentos de una nueva generación / Picture M exico City: Landmarks of a N ew Generation. Los Angeles-M éxico, G etty Conservation Institute-La Vaca Independiente, 1997.

Carlos Pellicer. Exposición homenaje, I8971997. M éxico, Instituto $\mathrm{N}$ acional de $\mathrm{Be}-$ Ilas Artes (M useo de Arte M oderno), 1997.

Cercanías distantes-D istant Relations. Arte chicano, irlandés y mexicano, textos críticos. Compilación de Trisha Ziff, introducción de Lucy R. Lippard, M éxico, Instituto N acional de Bellas Artes, 1997. so mujeres en la plástica de M éxico. Catálogo de la exposición de la Galería M etropolitana, M éxico, Instituto de Seguridad y Servicios Sociales de los T rabajadores del Estado-Universidad Autónoma M etropolitana, 1997.

Los colores del pensamiento de frontera a fron- tera. Expresiones plásicas de roo mujeres. M éxico, Universidad N acional Autónoma de M éxico, 1997.

Conde, T eresa del, T res maestros. Reflexiones sobre Bacon, M otherwell y Tamayo. M é xico, G rijalbo-U niversidad $\mathrm{N}$ acional Autónoma de M éxico, 1997.

Craven, D avid, D iego Rivera as Epic M odernist. N ueva York, G. K. H all, 1997.

$D$ ear Vocio: Photographs by Tina M odotti. Catálogo de la exposición, curada por Patricia Albers, de la U niversity Art $G$ allery de la University of $C$ alifornia en San D iego, textos de Kathleen Stoughton, Pasquale Verdicchio y Patricia Albers, La Jolla (California), University of California, 1997.

D iálogos insólitos: arte objeto. Catálogo de la exposición del M useo de Arte M oderno, Instituto $\mathrm{N}$ acional de Bellas Artes (M Useo de Arte M oderno)-Sociedad M exicana de Arte M oderno, 1997.

Diez y va un siglo. Libro conmemorativo de los diez años de la Semana Cultural Lésbico-G ay. M éxico, U niversidad $\mathrm{N}$ acional Autónoma de M éxico (M useo Universitario del Chopo-Coordinación de Difusión Cultural)-Círculo Cultural GayFondo $\mathrm{N}$ acional para la Cultura y las Artes, 1997.

Los ecos de M athias G oeritz. Catálogo de la exposición. Compilación de Ferruccio Asta, catálogo de la exposición del Antiguo Colegio de San Ildefonso, M éxico, Instituto Nacional de Bellas Artes-U niversidad $\mathrm{N}$ acional Autónoma de $\mathrm{M}$ éxico (Instituto de Investigaciones EstéticasCoordinación de Difusión Cultural-Antiguo Colegio de San IIdefonso)-Consejo $\mathrm{N}$ acional para la Cultura y las Artes-Instituto Goethe-Patronato de la Industria Alemana para la Cultura, 1997. 
Los ecos de M athias G oeritz. Ensayos y testimonios. Compilación de Ida Rodríguez Prampolini y Ferruccio Asta, M éxico, Instituto $\mathrm{N}$ acional de Bellas Artes-Consejo $\mathrm{N}$ acional para la Cultura y las ArtesUniversidad $\mathrm{N}$ acional Autónoma de M éxico (Instituto de Investigaciones Estéticas-Antiguo Colegio de San IIdefonSO), 1997.

Eduardo Cohen, 1939-I995. Los propósitos de la mirada. Edición de Rodolfo Rivera González, M éxico, Universidad Nacional Autónoma de M éxico, 1997.

Espinosa Campos, Eduardo, Carlos M érida, un artista integral. M éxico, Consejo $\mathrm{Na}$ cional para la Cultura y las Artes, 1997 (Círculo de Arte).

Fehr, Burkhar, Los tiranicidas, 0 ¿es posible erigir un monumento a la democracia? M éxico, Siglo Veintiuno, 1997 (Galería Abierta).

Fernando Leal Audirac: M-I3 Gallery. M éxiCo, Grupo Gráfico Romo, 1997.

García Barragán, Elisa, Carlos Pellicer en el espacio de la plástica. M éxico, Universidad $\mathrm{N}$ acional Autónoma de M éxico (D irección General de Artes Plásticas), 1997, 2 vols.

García Ponce, Juan, G arcía Ponce, 1933-1987. Pinturas, collages y gráfica. C atálogo de la exposición del M useo del Palacio de Bellas Artes, M éxico, Instituto $\mathrm{Na-}$ cional de Bellas Artes, 1997.

González M ello, Renato, José Clemente Orozco, la pintura mural mexicana. Mé xico, Consejo $\mathrm{N}$ acional para la $\mathrm{Cultura}$ y las Artes, 1997 (Círculo de Arte).

Guillermo Trujillo. M ito y metamorfosis. Catálogo de la exposición del M useo Rufino T amayo, edición de Carmen Alemán, M éxico, Embajada de PanamáConsejo Nacional para la Cultura y las
Artes-Instituto $\mathrm{N}$ acional de Bellas Artes, 1997.

I conografía de $D$ avid Alfaro Siqueiros. M éxiCo, Consejo $\mathrm{N}$ acional para la Cultura y las Artes-Instituto $\mathrm{N}$ acional de Bellas Artes ( $\mathrm{C}$ entro $\mathrm{N}$ acional de Investigación e Información de Artes Plásticas)-Fondo de Cultura Económica, 1997.

Jiménez, Víctor, Juan 0 'G orman. Principio y fin del camino. M éxico, Consejo $\mathrm{Na-}$ cional para la Cultura y las Artes, 1997 (Círculo de Arte).

J osé de Guimaraes: Serie M éxico. M éxico, M useo de Arte M oderno, 1997.

Juan Soriano: retrospectiva 1937-1997. M adrid, M useo N acional Centro de Arte Reina Sofía, 1997.

Kassner, Lily, D iccionario de escultores mexicanos de siglo Xx. M éxico, Consejo $\mathrm{Na}$ cional para la Cultura y las Artes, 1997.

The Life and Work of Luis Barragan. Traducción de Margaret E. Brooks, prólogo de Álvaro Siza, ensayos de José M aría Buendía Júlbez, Juan Palomar y Guillermo Eguiarte, fotografías de Sebastián Saldívar, N ueva York, Rizzoli, 1997.

M aestros del arte contemporáneo en la colección permanente del M useo Rufino Tamayo. M éxico, Instituto $\mathrm{N}$ acional de Bellas Artes, Fundación O lga y Rufino Tama yo, 1997.

M anifiesta 2. Cábula situacionista. Catálogo de exposición, M éxico, Instituto $\mathrm{N}$ acional de Bellas Artes (Ex Teresa Arte Alternativo), 1997.

M anuel Álvarez Bravo. Catálogo de la exposición en el M useum of M odern Art, texto de Susan Kismaric, N ueva York, M useum of M odern Art, 1997.

M auricio Cervantes: zona limial. M éxico, Instituto $\mathrm{N}$ acional de Antropología e 
H istoria (Coordinación N acional de Difusión), 1997.

M exiac, Adolfo, Mi paso por la xilografía. De la utilización de herramientas tradicionales al uso de aparatos automotivos y eléctricos. Discurso de ingreso a la Academia de Artes. Respuesta de Xavier M oyssén, M éxico, Academia de Artes, 1997.

$M$ exico N ow: Point of Departure / M éxico ahora: punto de partida. Catálogo de exposición, edición de Charles Fenton y N orma Roberts, fotografías de Juan José Márquez, traducción de Lorna Scott Fox y M aría Guerra, Columbus (O hio), O hio Arts Council, 1997.

$M$ odernity and the Architecture of M exico. Compilación de Edward R. Burian, Austin, U niversity of T exas, 1997.

M oreno Villarreal, Jaime, Ignacio Salazar. Catálogo de exposición, M éxico, Galería Arte Actual M exicano, 1997.

M ytlow, John V., Legorreta Arquitectos. M éxico, G ustavo G ili, 1997.

N oelle, Louise, Luis Barragán. Dilatazione emotiva degli spazi. Vol. 20. U niversale di Architettura Collana Diretta da Bruno Zevi. Torino, 1997.

${ }_{77}$ Salón de Arte Bancomer: pintura, escultura, arte objeto. M éxico, Fundación Cultural Bancomer, 1997.

$\mathrm{O}$ choa Vega, Alejandro, y Francisco $\mathrm{H}$ aroldo Alfaro Salazar, Espacios distantes... aún vivos. Las salas cinematográficas de la ciudad de M éxico. M éxico, U niversidad Autónoma M etropolitana-Xochimilco, 1997.

O rtiz M onasterio, Pablo, Idolatrías. Proyectos para esculturas monumentales. $M$ éxiCo, Instituto $\mathrm{N}$ acional de Bellas Artes (M useo de Arte Moderno)-Sociedad M exicana de Arte M oderno, 1997.

Passione per la vita: la rivoluzione dell'arte messicana nel $x x$ secolo. Catálogo de la exposición, curada por Alessandra Borghese y M iguel Ángel Corzo, del Castel dell'O vo, N ápoles, Leonardo Arte, 1997.

Pierre Alechinsky. Labor de imprenta de cinco décadas. $C$ atálogo de exposición, M éxi$\mathrm{Co}$, Consejo $\mathrm{N}$ acional para la Cultura y las Artes-Instituto $\mathrm{N}$ acional de Bellas Artes, 1997.

Portrait of a D ecade: D avid Alfaro Siqueiros, 1930-1940. Catálogo de la exposición del M useo N acional de Arte y el Santa Barbara M useum of Art, M éxico, Instituto $\mathrm{N}$ acional de Bellas Artes, 1997.

Raúl Anguiano: homenaje. M éxico, M iguel Ángel Porrúa, 1997.

Ricardo Legorreta, Architects. N ueva York, Rizzoli, 1997.

Rivera M arín, Guadal upe, Diego el rojo. M é xico, N ueva Imagen, 1997.

Rojo, Vicente, 0 bra sobre papel y gran escenario primitivo. Catálogo de exposición, $\mathrm{M}$ adrid, $\mathrm{M}$ useo $\mathrm{N}$ acional $\mathrm{C}$ entro de $\mathrm{Ar}$ te Reina Sofía, 1997.

Rossi, Alejandro, Teodoro G onzález de León. Juegos de magia. M éxico, Consejo $\mathrm{Na}$ cional para la Cultura y las Artes, 1997 (Círculo de Arte).

Schjetnan, M ario, y José Luis Pérez, Arquitectura, ciudad y naturaleza. M éxico, Consejo $\mathrm{N}$ acional para la Cultura y las Artes, 1997.

Sebastián ante la crítica. Compilación de Roberto Vallarino, M éxico, El Equilibrista, 1997.

Segunda Bienal de M onterrey. Pintura, escultura e instalación 1994. M éxico, M useo de M onterrey, 1997.

Sociedad M exicana de Acuarelistas, 1964 1997. M éxico, Sociedad M exicana de Acuarelistas, 1997.

Talavera. T radición de vanguardia, artistas 
contemporáneos. M éxico, M useo Amparo, 1997.

Taller de Gráfica Popular: 60 años. M éxico, Instituto $\mathrm{N}$ acional de Bellas Artes, 1997.

Las transgresiones al cuerpo. Catálogo de la exposición del Museo de Arte Carrillo Gil, M éxico, Instituto $\mathrm{N}$ acional de $\mathrm{Be}$ llas Artes (M useo de Arte Carrillo Gil), 1997.

Tres generaciones: Rodolfo M orales, Francisco Toledo, Julio Galán. M éxico, Confederación de Educadores Americanos, 1997.

Villoro, Juan, M anuel Felguérez. El límite de una secuencia. M éxico, Consejo $\mathrm{N}$ acional para la Cultura y las Artes, 1997 (Círculo de Arte).

Zamora Betancourt, Lorena, O Iga Coła, un espíritu sensible. M éxico, Consejo $\mathrm{Na}$ cional para la Cultura y las Artes, 1997 (Círculo de Arte).

Zárraga. M éxico, Bital-Américo Arte, 1997.

\section{Folklore y arte popular}

Arte popular mexicano. Cinco siglos. Catálogo de la exposición del Antiguo Colegio de San Ildefonso, texto de Juan Coronel Rivera, M éxico, U niversidad $\mathrm{N}$ acional Autónoma de M éxico, 1997.

Cerámica inglesa en M éxico, M éxico, Artes de M éxico, 1997.

Lacas mexicanas. M éxico, M useo Franz $M$ ayer-Artes de M éxico, 1997.

M apelli M ozzi, Carlota, Xuchiles o ramilletes. Adornosflorales de siglo XVI al Xx en $M$ éxico. M éxico, Ruf O rganización, 1997.

M éxico pictórico y artesano: arte contemporáneo, arte popular mexicano. Catálogo de exposición, Puebla, M useo Amparo, 1997.
O ikion Solano, Verónica, M anos michoacanas. M éxico, El Colegio de M ichoacán, 1997.

Salvo, D ana, H ome Altars of M exico. Edición de Ramón $A$. Gutiérrez, textos de Ramón A. Gutiérrez, Salvatore Scalora, William H. Beezley y Amalia M esaBains, Albuquerque, University of $\mathrm{New}$ M exico, 1997.

\section{Cine, música y artes escénicas}

Cuarteto H einer M uller. M éxico, Instituto $\mathrm{N}$ acional de Bellas Artes, 1997.

Elías, M anuel de, U na breve visión panorámica de la ópera en M éxico. M éxico, Aca demia de Artes, 1997.

Ibarra, Federico, El compositor como portador del tiempo. Discurso de ingreso a la Academia de Artes. Respuesta de M ario Lavista, M éxico, Academia de Artes, 1997.

0 axaca en Eduardo $M$ ata. M éxico, Instituto 0 axaqueño de las $C$ ulturas, 1997.

Vega Alfaro, Eduardo de la, Del muro a la pantalla. S. M. Eisenstein y el arte pictórico mexicano. Guadalajara-T oluca, U niversidad de Guadalajara-I nstituto M exiquense de Cultura, 1997.

\section{H istoriografía}

Acha, Juan, L os conceptos esenciales de las artes plásticas. M éxico, C oyoacán, 1997.

Breton, André, U n listón alrededor de una bomba. U na mirada sobre el arte mexicano. M éxico, Instituto $\mathrm{N}$ acional de $\mathrm{Be}$ Ilas Artes, 1997.

Sánchez Vázquez, Adolfo, Cuestiones estéticas y artísticas contemporáneas. M éxico, 
Fondo de Cultura Económica, 1997.

Rivero Lake, Rodrigo, La visión de un anticuario. Presentación de Álvaro Mutis, texto de Roberto Vallarino, fotografías de $M$ ichel Z abe, edición de $M$ arcela Aguilar y M aya de Rivero Lake, M éxico, Américo Arte, 1997.

W estphalen, Emilio Adolfo, Escritos varios sobre arte y poesía. M éxico, Fondo de Cultura Económica, 1997 (Tierra Firme). W estheim, Paul, Pensamiento artístico y creación: ayer y hoy. M éxico, Siglo Veintiuno, 1997.

\section{Varios}

Arte y Espacio. XIX Coloquio Internacional de Historia del Arte. Compilación de Ó scar O lea, M éxico, U niversidad $\mathrm{N}$ acional Autónoma de M éxico (Instituto de Investigaciones Estéticas), 1997.

La catedral de M éxico. Problemática, restauración y conservación en el futuro. $2^{0}$. CoIoquio del Seminario de Estudios del Patrimonio Artístico. Compilación de $M$ artha Fernández, M éxico, U niversidad $\mathrm{N}$ acional Autónoma de M éxico (Instituto de Investigaciones Estéticas), 1997 (Estudios de Arte y Estética, 40).

El centro histórico ayer, hoy y mañana. Compilación de $C$ ristina Barros, M éxico, Instituto $\mathrm{N}$ acional de Antropología e H istoria, 1997.

De M éxico y de la humanidad. M éxico, AxA, 1997.

Especulación y patrimonio. $4^{0}$. Coloquio del Seminario de Estudios del Patrimonio Artístico, Conservación, Restauración y $D$ efensa. Compilación de Enrique $X$. de Anda Alanís, M éxico, Universidad $\mathrm{N}$ acional Autónoma de M éxico (Instituto de Investigaciones Estéticas), 1997.

$\mathrm{H}$ istoria del arte de 0 axaca. O axaca, M éxico, Instituto $O$ axaqueño de las $C$ ulturas, 1997, 3 vols.

Levitt, $\mathrm{H}$ elen, M exico City. Ensayo de James O les, N ueva York, Center for D ocumentary Studies-W. W . N orton, 1997.

$M$ exican M asterpieces from the Bernard and Edith Lewin Collection: Los Angeles County M useum of Art, N ovember 23, 1997February 16 , 1998 / O bras maestras mexicanas de la colección de Bernard y Edith Lewin, M useo de Arte del Condado de LoS Ángeles, desde el 23 de noviembre de 1997 hasta e $I_{1} 6$ de febrero de 1998 . Catálogo de exposición, Los Angeles, Los Angeles County M useum of Art, 1997.

M ijares Bracho, Carlos, San Ángel. M éxico, Clío, 1997.

Picinelli, Filippo, Los cuerpos celestes. Libro I: el libro simbólico. M éxico, El Colegio de M ichoacán, I997.

Real del M onte: el esplendor de ayer para siempre. M éxico, Gobierno del Estado de Hidalgo, 1997.

Reyna, $\mathrm{M}$ aría del $\mathrm{C}$ armen, $\mathrm{H}$ aciendas en $\mathrm{el}$ sur de la ciudad de M éxico. M éxico, Instituto $\mathrm{N}$ acional de Antropología e $\mathrm{H}$ istoria, 1997.

La sociedad civil frente al patrimonio cultural. $3^{\text {er }}$. Coloquio del Seminario de Estudios del Patrimonio Artístico, Conservación, Restauración y D efensa. Compilación de Francisco Vidargas, M éxico, Universidad $\mathrm{N}$ acional Autónoma de M éxico (Instituto de Investigaciones Estéticas), 1997 (Estudios de Arte y Esté tica, 44).

Subasta amigos del M useo de Arte M oderno. $M$ iércoles, 29 de octubre. M éxico, Galerías Louis C. M orton, 1997.

Subasta del Ático. M éxico, Galerías Louis C. 
M orton, 1997.

Subasta del Ático. Antigüedades, sábado I8 de octubre. M éxico, Galerías Louis C. M orton, 1997.

Subasta del Ático. Antigüedades, sábado 27 de septiembre de 1997. M éxico, G alerías Louis C. M orton, 1997.

Subasta general en M onterrey, miércoles 22 de octubre. M éxico, Galerías Louis M orton, 1997.

Subasta de libros, acciones, fotografía y postales. M éxico, Galerías Louis C. M orton, 1997.

Subasta tradicional de antigüedades y juguetes de colección. M éxico, Galerías Louis C.
M orton, 1997.

Temas y problemas, $I^{\text {er }}$. Coloquio de Seminario de Estudios del Patrimonio Artístico, Conservación, Restauración y $D$ efensa. M éxico, Universidad $\mathrm{N}$ acional Autónoma de M éxico (Instituto de Investigaciones Estéticas), 1997.

Tovar de Teresa, Guillermo, Repertorio de artistas en M éxico. Artes pláticas y decorativas (P-Z). M éxico, Bancomer, 1997, vol. 3 .

Zavala, Silvio, En defensa del Paseo de la Reforma. U niversidad I beroamericana (D epartamento de Arte), 1997. 